doi:10.1017/\$1041610219000656

\section{Sharing is Caring: WhatsApp as a method of disseminating dementia knowledge to elderly Chinese Americans}

As prevalent as dementia is within the worldwide aging population, it remains a stigmatized disease within the Chinese community (Woo and Mehta, 2017). The lack of information suitable for the Chinese speaking population acts as a major barrier in disease detection and treatment. Previous studies have shown that YouTube has the potential to deliver dementia education to elderly Chinese populations (Lam et al., 2017; Zheng and Woo, 2017). Our study examines how the Chinesespeaking public shares dementia education videos amongst themselves through various social media platforms.

A board-certified psychiatrist was invited onto a Chinese television station for an educational talk show in Cantonese, covering topics on dementia background, prevention, and management. The real-time recording was then uploaded to YouTube as two 25-minute videos. Using YouTube Analytics, the data extrapolated included parameters such as age of viewers, number of views, average length of videos watched, traffic sources, and number of times the video was shared broken down by each social media platform.

From March 2014 to March 2019, data captured from the two YouTube videos showed that the video was viewed 29,658 times by Chinese speaking viewers aged 65 and older. The average watch time on any mobile device was 6.7 minutes, while the average watch time viewed through the WhatsApp mobile app was 4.9 minutes. Of the traffic sources, 5056 views $(17.0 \%)$ were externally sourced, with the top two communication platforms being WhatsApp and Facebook. Videos viewed through WhatsApp accounted for $2.9 \%(n=864)$ of total views while Facebook accounted for $0.2 \%$ $(\mathrm{n}=60)$. The video was shared 879 total times with $64.3 \%(n=565)$ of videos shared through WhatsApp, 4.1\% $(\mathrm{n}=36)$ through Facebook, and 31.6\% $(\mathrm{n}=278)$ shared through other methods. WhatsApp was utilized more for its sharing capabilities than for its viewing functions $\left(64.3 \%\right.$ vs $1.9 \% ; x^{2}=7193$, $\mathrm{df}=1, \mathrm{p}<.01)$.
With over 500 million monthly active users sharing 100 million videos daily, WhatsApp is one of the most widely used cross-platform messaging services for sending text messages, images, and other media (Chowdhry, 2014). WhatsApp has been used in classrooms to stimulate discussion and foster active learning (Bouhnik et al., 2014), but it has never been studied as a method of disseminating dementia knowledge. Our study found that WhatsApp was the preferred method used by elderly Chinese Americans to share dementia knowledge with others. Although a multifunctional application, the studied sample used WhatsApp more for its sharing capabilities than for its viewing purposes. The average watch time on WhatsApp and on any mobile device was approximately 5 and 6.7 minutes, respectively. This suggests that medical videos should be limited in duration to keep viewers engaged.

Our study demonstrated that WhatsApp holds potential as a significant vehicle for educating the Chinese-speaking public regarding dementia. Future research should analyze how WhatsApp can expand outreach and cater to populations of interest. Studying how groups of people choose to communicate and spread information amongst themselves is a new and exciting approach to patient education in a time when technology and healthcare is at its peak.

\section{References}

Bouhnik, D., Deshen, M. and Gan, R. (2014). WhatsApp goes to school: mobile instant messaging between teachers and students. Fournal of Information Technology Education: Research, 13, 217-231. doi: 10.1016/j.ajp.2016 .12 .002 .

Chowdhry, A. (2014). WhatsApp Hits 500 Million Users. Forbes.com. https://www.forbes.com/sites/amitchowdhry/ 2014/04/22/whatsapp-hits-500-million-users/ \#51a75d14702a; accessed 23 January 2019.

Lam, N. H. T., Tsiang, J. T. H. and Woo, B. K. P. (2017). Exploring the role of YouTube in disseminating psychoeducation. Academic Psychiatry: The Fournal of the American Association of Directors of Psychiatric Residency Training and the Association for Academic Psychiatry, 41, 819-822. doi: 10.1007/s40596-017-0835-9.

Woo, B. K. and Mehta, P. (2017). Examining the differences in the stigma of dementia and diabetes among Chinese Americans. Geriatrics \& Gerontology International, 17, 760-764. doi: 10.1111/ggi.12782. 
Zheng, X. and Woo, B. K. P. (2017). E-mental health in ethnic minority: a comparison of YouTube and talk-based educational workshops in dementia. Asian fournal of Psychiatry, 25, 246-248. doi: 10.1016/j.ajp.2016.12.002.

Becky Nguyen ${ }^{1}$ and Benjamin K. P. Woo ${ }^{2}$

${ }^{1}$ Western University of Health Sciences, Pomona, CA, USA

${ }^{2}$ Olive View - UCLA Medical Center, Sylmar, CA, USA
Correspondence should be addressed to: Becky Nguyen, Western University of Health Sciences, 309 E. 2nd St., Pomona, CA 91766, USA. Email: beckynguyen91@ gmail.com. 